\title{
A NOTE ON THE NORMAL GENERATION OF AMPLE LINE BUNDLES ON AN ABELIAN SURFACE
}

\author{
AKIRA OHBUCHI \\ (Communicated by Louis J. Ratliff, Jr.)
}

\begin{abstract}
Let $L$ be an ample line bundle on an abelian surface $A$. We prove that the four conditions: (1) $L$ is base point free, (2) $L$ is fixed component free, (3) $L^{\otimes 2}$ is very ample, (4) $L^{\otimes 2}$ is normally generated, are equivalent if $\left(L^{2}\right)>4$. Moreover we prove that $L^{\otimes 2}$ is not normally generated if $\left(L^{2}\right)=4$.
\end{abstract}

\section{INTRODUCTION}

Let $B$ be an abelian variety defined over an algebraically closed field, and let $M$ be an ample line bundle on $B$. The theorem of Lefschetz says that $M^{\otimes n}$ is very ample if $n \geq 3$. If a polarized abelian variety $(B, M)$ is isomorphic to $\left(B_{1} \times B_{2}, \mathscr{O}\left(\Theta_{1} \times B_{2}+B_{1} \times D_{2}\right)\right)$ where $\Theta_{1}$ is a principal polarization of $B_{1}, \operatorname{dim}\left(B_{1}\right)>0$, and $\operatorname{dim}\left(B_{2}\right) \geq 0$, then $M^{\otimes 2}$ is not very ample. Therefore the Lefschetz theorem gives a best possible condition for the very ampleness of $M^{\otimes n}$. The above example, however, is the only example for which $M^{\otimes 2}$ is not very ample (see Ohbuchi [3]). The condition that $M^{\otimes n}$ is normally generated is also given (see Koizumi [1], Sasaki [5], Sekiguchi [6-8]) and $n=3$ is best possible in this case too. As for $M^{\otimes 2}$, if $M$ is base point free then $M^{\otimes 2}$ is normally generated (see Ohbuchi [4]). In this paper we consider the difference between this very ample condition and this normally generated condition. The result is the following:

Theorem. Let $L$ be an ample line bundle on an abelian surface $A$ defined over an algebraically closed field with characteristic 0 . If $\left(L^{2}\right)>4$ then the following conditions are equivalent:

(1) $L$ is fixed component free,

(2) $L$ is base point free,

(3) $L^{\otimes 2}$ is very ample,

(4) $L^{\otimes 2}$ is normally generated.

If $\left(L^{2}\right) \leq 4$ then the above conditions are not equivalent. If $\left(L^{2}\right)=2$ then $L^{\otimes 2}$ is not very ample, and therefore is not normally generated. In the last part of this paper, we prove that $L^{\otimes 2}$ is not normally generated if $\left(L^{2}\right)=4$.

Received by the editors February 7, 1991 and, in revised form, May 23, 1991.

1991 Mathematics Subject Classification. Primary 55R25. 


\section{PROOF OF THE THEOREM}

To prove the above theorem, we prepare several lemmas. Throughout this paper, we assume that $L$ is an ample line bundle on an abelian surface $A$ defined over an algebraically closed field.

Lemma 1. Let $B$ be an abelian variety and $M$ be an ample line bundle on $B$. $M^{\otimes 2}$ is not very ample if and only if the polarized variety $(B, \underline{M})$ is isomorphic to $\left(B_{1} \times B_{2}, \mathscr{O}\left(D_{1} \times B_{2}+B_{1} \times D_{2}\right)\right)$ where $B_{1}$ and $B_{2}$ are abelian varieties with $\operatorname{dim} B_{1}>0$ and $D_{1}$ and $D_{2}$ are ample divisors with $\operatorname{dim} \Gamma\left(B_{1}, \mathscr{O}\left(D_{1}\right)\right)=1$.

Proof. See Ohbuchi [3]. Q.E.D.

Lemma 2. $L$ is fixed component free if and only if $L^{\otimes 2}$ is very ample.

Proof. We may assume that $\left(L^{2}\right)>2$. We assume that $L$ has a fixed component. Let $F$ be a fixed part of a complete linear system $|L|$, and put $|L|=|M|+F$. If $\left(M^{2}\right)>0$ then $M$ is ample. Moreover, $\underline{L} \simeq \underline{M} \otimes \mathscr{O}(F)$ implies $\left(L^{2}\right)=\left((M+F)^{2}\right)$. Therefore $(M \cdot F)=\left(F^{2}\right)=0$. This is a contradiction because $M$ is an ample line bundle. Hence $\left(M^{2}\right)=0$. Moreover, we can see that $\left(F^{2}\right)=0$. In fact, we assume that $\left(F^{2}\right)>0$. In this case, if $x \in K(L)=\left\{x \in A, T_{x}^{*} L \simeq L\right\}$ then $x \in K(F)=\left\{x \in A, T_{x}^{*} F\right.$ is linearly equivalent to $F\}=\left\{x \in A ; T_{x}^{*} F=F\right\}$. By $\left(F^{2}\right)>0$, the order of $K(F)=$ $\left(\operatorname{dim}_{k} \Gamma(A, \mathscr{O}(F))\right)^{2}=1$. Hence $\left(L^{2}\right)=2$. This is a contradiction. Therefore $(A, L)$ is isomorphic to $\left(A_{1} \times A_{2}, \mathscr{O}\left(D_{1} \times A_{2}+A_{1} \times D_{2}\right)\right)$ where $A_{1}=A / K^{\circ}(M)$, $A_{2}=A / K^{\circ}(F), K^{\circ}(M)=$ the connected component of $K(M) \ni 0, K^{\circ}(F)=$ the connected component of $K(F) \ni 0$, and $D_{1}, D_{2}$ are divisors corresponding to $M, F$. As $F$ is a fixed part, $\operatorname{dim}_{k} \Gamma\left(A_{2}, \mathscr{O}\left(D_{2}\right)\right)=1$. Therefore, we obtain this lemma by Lemma 1. Q.E.D.

Lemma 3. Let $B$ be an abelian variety of dimension $g$, and let $M$ be an ample line bundle whose base points are at most a finite set. If $\left(M^{g}\right)>(g !)^{2}$ then $M$ is base point free.

Proof. We assume that the set of all base points $B s|M|$ is not empty. Let $p \in B s|M|$. We consider the set $K(M)=\left\{x \in B ; T_{x}^{*} M \simeq M\right\}$. By the definition of $K(M), p+K(M) \subset B s|M|$. Hence $\left(\left(M^{g}\right) / g !\right)^{2} \leq\left(M^{g}\right)$ because the order of $K(M)$ is $\left(\left(M^{g}\right) / g !\right)^{2}$. Therefore this lemma is obtained. Q.E.D.

Lemma 4. Let $M$ be a symmetric ample line bundle on a g-dimensional abelian variety $B$. Let $\alpha, \beta \in B$ (= the dual abelian variety) and let $u \in B$ be an element such that $2 u=\alpha-\beta$. Then $\Gamma\left(B, \underline{M}^{2} \otimes \mathscr{P}_{\alpha}\right) \otimes \Gamma\left(B, \underline{M}^{2} \otimes \mathscr{P}_{B}\right) \rightarrow$ $\Gamma\left(B, \underline{M}^{4} \otimes \mathscr{P}_{\alpha+\beta}\right)$ is surjective if and only if $\eta+u$ is not contained in $\phi_{M}(B s|M|)$ for every $\eta \in B[2]=\{x \in B ; 2 x=0\}$ where $\mathscr{P}$ is a Poincaré bundle and $\phi_{M}: B \rightarrow B$ is a dual map.

Proof. See Ohbuchi [4]. Q.E.D.

Proof of the theorem. The implications $(2) \rightarrow(1)$ and $(4) \rightarrow(3)$ are obvious. By Lemma 2, the conditions (1) and (3) are equivalent. By Lemma 4, (2) $\rightarrow(4)$ is obtained. By Lemma $3,(1) \rightarrow(2)$ is obtained if $\left(L^{2}\right)>4$. Q.E.D. 
If $\left(L^{2}\right)=2$ then $L$ is a principal polarization. Therefore $L^{\otimes 2}$ is not very ample, so $L^{\otimes 2}$ is not normally generated. If $\left(L^{2}\right)=4$ then the above equivalence does not hold because $L$ always has base points. But in this case, as for $L^{\otimes 2}$, we can show that $L^{\otimes 2}$ is never normally generated and that $L^{\otimes 2}$ is very ample provided that $L$ is fixed component free. We check this result.

Lemma 5. If $\left(L^{2}\right)=4$ and $L$ is fixed component free, then a general $C \in|L|$ is smooth.

Proof. By Bertini's theorem (see Zariski [9]), a general member $C \in|L|$ is smooth at $p \in C-B s|L|$. Let $p \in B s|L|$. Since $p+K(L) \subset B s|L|$ and the order of $B s|L| \leq 4$, it follows that $B s|L|$ is a 4-point set. For every $p \in B s|L|$, the intersection multiplicity at $p=(L \cdot C)_{p} \geq 1$. Hence $C$ is smooth at $p \in B s|L|$ because $\left(C^{2}\right)=4$. Q.E.D.

Lemma 6. $L^{\otimes 2}$ is not normally generated.

Proof. We may assume that $L$ is fixed component free. We also assume that $B s|L| \ni 0$. As $K(L)=B s|L|, B s|L|$ is contained in $A$ [2]. Let $C$ be a smooth member of $|L|$. Let $l: A \rightarrow A$ be a morphism defined by $l(x)=-x$. In this case we obtain that $\imath^{*} C=C$. Because the condition $l^{*} C \neq C$ implies that $C$ and $l^{*} C$ have a same tangent direction at every $p \in B s|L|$, it follows that $(d l)_{p}: T_{p}(A) \rightarrow T_{p}(A)$ is multiplication by -1 . Therefore $\left(C \cdot l^{*} C\right)_{p} \geq 2$. This is a contradiction. Hence $C$ is a symmetric divisor on $A$. By Lemma 4, we obtain this lemma. Q.E.D.

\section{REFERENCES}

1. S. Koizumi, Theta relations and projective normality of abelian varieties, Amer. J. Math. 98 (1976), 868-889.

2. D. Mumford, Abelian varieties, Oxford Univ. Press, New York, 1970.

3. A. Ohbuchi, Some remarks on ample line bundles on abelian varieties, Manuscripta Math. 57 (1987), 225-238.

4. __ A note on the normal generation of ample line bundles on abelian varieties, Proc. Japan Acad. Ser. A Math. Sci. 64 (1988), 119-120.

5. R. Sasaki, Theta relations and their applications in abstract geometry, Sci. Rep. Tokyo Kyoiku Daigaku 381 (1977), 138-160.

6. T. Sekiguchi, On projective normality of abelian varieties, J. Math. Soc. Japan 28 (1976), 307-322.

7. $\frac{}{727}$, On projective normality of abelian varieties. II, J. Math. Soc. Japan 29 (1977), 709-

8. __ On the cubics defining abelian varieties, J. Math. Soc. Japan 30 (1977), 703-721.

9. O. Zariski, Introduction to the problem of minimal models in the theory of algebraic surfaces, Math. Soc. Japan (1958).

Department of Mathematics, Faculty of Education, Yamaguchi University, YAMAGUCHI-SHI, YAMAGUCHI 753, JAPAN 